\title{
Systems Consolidation Requires Postlearning Activation of NMDA Receptors in the Medial Prefrontal Cortex in Trace Eyeblink Conditioning
}

\author{
Kaori Takehara-Nishiuchi, ${ }^{1}$ Kazuhito Nakao, ${ }^{2}$ Shigenori Kawahara, ${ }^{1}$ Norio Matsuki, ${ }^{2}$ and Yutaka Kirino ${ }^{1}$ \\ ${ }^{1}$ Laboratory of Neurobiophysics and ${ }^{2}$ Laboratory of Chemical Pharmacology, School of Pharmaceutical Sciences, The University of Tokyo, Tokyo \\ 113-0033, Japan
}

\begin{abstract}
The importance of the hippocampus in declarative memory is limited to recently acquired memory, and remotely acquired memory is believed to be stored somewhere in the neocortex. However, it remains unknown how the memory network is reorganized from a hippocampus-dependent form into a neocortex-dependent one. We reported previously that the medial prefrontal cortex (mPFC) is important for this neocortex-dependent remote memory in rat trace eyeblink conditioning. Here, we investigate the involvement of NMDA receptors in the $\mathrm{mPFC}$ in this reorganization and determine the time window of their contribution using chronic infusion of an antagonist into the $\mathrm{mPFC}$, specifically during the postlearning consolidation period. The rats with blockade of the mPFC NMDA receptors during the first 1 or 2 weeks after learning showed a marked impairment in memory retention measured 6 weeks after learning, but relearned normally with subsequent conditioning. In contrast, the same treatment had no effect if it was performed during the third to fourth weeks or during the first day just after learning. The specificity of NMDA receptor blockade was confirmed by the reduced long-term potentiation in the hippocampal-prefrontal pathway in these rats. These results suggest that successful establishment of remotely acquired memory requires activation of NMDA receptors in the $\mathrm{MPFC}$ during at least the initial week of the postlearning period. Such NMDA receptor-dependent processes may mediate the maturation of neocortical networks that underlies permanent memory storage and serve as a way to reorganize memory circuitry to the neocortex-dependent form.
\end{abstract}

Key words: classical conditioning; eyeblink; retention; amnesia; hippocampus; rat; APV; prelimbic; retrieval

\section{Introduction}

The phenomenon of temporally graded retrograde amnesia has interested scientists for more than 100 years (Squire, 1992). In studies of both humans and experimental animals with hippocampal damage, memory loss is often graded within long-term memory, such that a remotely acquired memory is spared relative to a recently acquired one. These findings suggest that memories are gradually established in extrahippocampal networks through a process of memory consolidation (Squire and Alvarez, 1995; Knowlton and Fanselow, 1998) (but see Nadel and Moscovitch, 1997). This type of consolidation is called "systems consolidation" in the recent literature, to distinguish it from other processes of cellular consolidation by which short-term synaptic modifications induced by experience are stabilized and become

\footnotetext{
Received 0ct. 15, 2005; revised March 24, 2006; accepted March 24, 2006.

This work was supported by Grants 16390015 and 17209002 from the Ministry of Education, Culture, Sports, Science and Technology of Japan, and Core Research for Evolution Science and Technology of the Japan Science and Technology Agency. K.T. is the recipient of a Fellowship for Young Scientists from the Japan Society for the Promotion of Science.

Correspondence should be addressed to Kaori Takehara-Nishiuchi, Laboratory of Neurobiophysics, School of Pharmaceutical Sciences, The University of Tokyo, 7-3-1 Hongo, Bunkyo-ku, Tokyo 113-0033, Japan. E-mail: ktakehara@mayqueen.f.u-tokyo.ac.jp.

DOI:10.1523/JNEUROSCI.4381-05.2006

Copyright $\odot 2006$ Society for Neuroscience $\quad$ 0270-6474/06/265049-10\$15.00/0
}

enduring changes in connectivity between neurons (Debiec et al., 2002).

We have identified the medial prefrontal cortex $(\mathrm{mPFC})$ as an important component of the extrahippocampal network that mediates remotely acquired memory, because a postlearning lesion in the mPFC severely impairs retention of remotely acquired memory, but only marginally impairs recently acquired memory in rat trace eyeblink conditioning (Takehara et al., 2003). This learning is a hippocampus-dependent variation of classical eyeblink conditioning, a well characterized model of associative learning (Thompson and Kim, 1996). Because a hippocampal lesion preferentially eliminates recently acquired memory in this task (Kim et al., 1995; Takehara et al., 2002, 2003), these results suggest the possibility that the brain circuitry mediating memory is gradually reorganized from a form involving the hippocampus into one involving the mPFC during the course of systems consolidation. However, little is known about how this reorganization is accomplished.

One possible mechanism for this reorganization is that neocortical networks gradually mature, possibly through repeated reactivations of neocortical memory modules by the hippocampus (McClelland et al., 1995; Squire and Alvarez., 1995). According to this view, some kinds of plastic changes should occur in the mPFC, in which memory is presumed to be consolidated, and blockade of these changes should interfere with establishment of 
remotely acquired memory. Therefore, the objective of this study is to identify the mechanism by which the mPFC contributes to the reorganization of memory networks, and to determine the time window of its contribution. To this end, we focused on NMDA receptors in the $\mathrm{mPFC}$ because NMDA receptors are involved in the consolidation process in the hippocampus (Shimizu et al., 2000) and NMDA receptor-dependent neural plasticity has been reported in in vitro slice preparations (Hirsch and Crepel, 1991) and in the hippocampal-prefrontal pathway in vivo (Laroche et al. 1990; Jay et al., 1995). Chronic focal infusion of a neuroactive drug with osmotic pumps during the consolidation period offers an opportunity to manipulate neuronal processes in the $\mathrm{MPFC}$ without affecting any processes of acquisition or retrieval. We show that such chronic infusion of the NMDA receptor antagonist D-2-amino-5-phosphonovaleric acid (DAPV) into the mPFC during the initial 1 or 2 weeks of the postlearning period severely impairs consolidation of memory in neocortical networks in trace eyeblink conditioning, although infusion during the second 2 weeks or first day does not impair consolidation.

\section{Materials and Methods}

Subjects. The subjects were 122 male Wistar rats (Japan SLC, Hamamatsu, Shizuoka, Japan) weighing 210-270 g (9 weeks old) at the time of the first surgery. The animals were housed in standard plastic cages in a colony room with a $12 \mathrm{~h}$ light/dark cycle. Water and food were available ad libitum. All experiments were performed in accordance with the guidelines established by the Institutional Animal Investigation Committee at the University of Tokyo and the United States National Institutes of Health Guide for the Care and Use of Laboratory Animals. Every effort was made to optimize comfort and to minimize the use of animals.

Surgery for acute infusion. Twelve rats received two kinds of surgery in series: implanting electrodes before the conditioning and implanting the guide cannulas and electrodes before the reconditioning. In the first surgery, four Teflon-coated stainless-steel wires (\#7910; A-M Systems, Carlsborg, WA) were implanted subcutaneously in the left upper eyelid under anesthesia with ketamine $(87 \mathrm{mg} / \mathrm{kg}$, i.p.; Sankyo, Tokyo, Japan) and xylazine (13 mg/kg, i.p.; Bayer, Tokyo, Japan). These wires were soldered to the pins of a connector, which was secured to the skull anterior to bregma with dental acrylic resin and stainless-steel screws. In the second surgery, performed at least 1 week before the re-conditioning, the trained animals were anesthetized in the same way as in the first surgery and their heads were placed in stereotaxic frames (Narishige, Tokyo, Japan) with the skull surface in the horizontal plane. After the connector with the electrodes was removed and the skull was exposed by incision along the midline, small holes ( $0.8 \mathrm{~mm}$ diameter) were drilled on each side of the skull at the following coordinates (in $\mathrm{mm}$ from bregma): anteroposterior $(\mathrm{AP})+3.2$, mediolateral $(\mathrm{ML}) \pm 1.9$. Two single guide cannulas (26 gauge; Plastic One, Roanoke, VA) were implanted at an angle of $\pm 20^{\circ}$ through each hole and their tips were directed to the following coordinates: $\mathrm{AP}+3.2, \mathrm{ML} \pm 0.8$, dorsoventral (DV) -2.61 to the skull surface. Because the tip of the infusion cannula protruded into the prelimbic area $1 \mathrm{~mm}$ from the guide cannula, the final infusion positions were at the following coordinates: $\mathrm{AP}+3.2, \mathrm{ML} \pm 0.7, \mathrm{DV}$ -3.5 . The guide cannulas were fixed on the skull with dental cement and small stainless-steel screws. Stainless-steel stylets, which extended $1 \mathrm{~mm}$ beyond the tip of the guide cannulas, were placed inside the guide cannulas to prevent occlusion. Then, new electrodes were implanted again.

Surgery for chronic infusion. Eighty-five rats received four kinds of surgery in series: implanting electrodes before the conditioning, implanting the infusion cannulas connected to osmotic pumps, removing the osmotic pumps, and implanting electrodes before the reconditioning. In the first surgery, stainless-steel wires were implanted in the same way as that mentioned in the surgery for acute infusion. In the second surgery, in the same way as acute infusion, small holes $(0.8 \mathrm{~mm}$ diameter $)$ were drilled on each side of the skull at the following coordinates (in $\mathrm{mm}$ from bregma): AP $+3.2, \mathrm{ML} \pm 1.96$. Two single infusion cannulas [28 gauge (Plastic One) or Alzet Brain Infusion Kit I (Durect, Cupertino, CA)] were implanted at an angle of $\pm 20^{\circ}$ through each hole and their tips were directed to the following coordinates: $\mathrm{AP}+3.2, \mathrm{ML} \pm 0.9, \mathrm{DV}-3.9$ to the skull surface. These pumps were connected via flexible polyethylene tubing to osmotic pumps (Alzet mini-osmotic pump, model 2002; Durect), which contained artificial CSF (aCSF) or D-APV (4 mm; Sigma-RBI, Tokyo, Japan). We chose this concentration of D-APV because it was the minimum concentration needed to block conditioned response (CR) acquisition sufficiently when it was infused during acquisition in our preliminary study. The infusion cannulas were fixed on the skull with dental cement and small stainless-steel screws. At the end of the infusion period, the implanted pumps were removed under the same anesthesia. At least $4 \mathrm{~d}$ before the reconditioning, the new electrodes were implanted again. The animals were injected with gentamycin sulfate $(8 \mathrm{mg} / \mathrm{kg}$, i.p.; Schering-Plough, Osaka, Japan) after the latter three surgeries and warmed until they moved spontaneously.

Conditioning procedure. Four cylindrical Plexiglas containers $(15 \mathrm{~cm}$ in diameter and $25 \mathrm{~cm}$ high) were placed in a sound- and light-attenuated chamber and one rat was placed in each container. A lightweight cable was connected to the connector pins secured on the animal's head. The conditioned stimulus (CS) was a $350 \mathrm{~ms}$ tone $(5 \mathrm{kHz}, 85 \mathrm{~dB})$ with a rise/fall time of $10 \mathrm{~ms}$; it was delivered from a speaker $(16.5 \mathrm{~cm}$ in diameter) placed above the containers. The unconditioned stimulus (US) was a $100 \mathrm{~ms}$ periorbital shock ( $100 \mathrm{~Hz}$ square pulses) delivered through a pair of electrodes implanted in the left upper eyelid; its intensity was carefully calibrated to give the minimal current required to elicit an eyeblink/head-turn response, and adjusted daily for each rat. A stimulusfree trace interval of $500 \mathrm{~ms}$ was interposed between the end of the CS and the US onset. The CR was monitored through electromyographic (EMG) activity recorded with another pair of implanted electrodes. Two to $4 \mathrm{~d}$ after the first surgery, spontaneous eyeblinking was recorded for two session days in the same way as during the conditioning session, except that no stimuli were presented. We started the conditioning beginning 4-6 d after the first surgery. A daily session consisted of 100 trials grouped into 10 blocks, which included nine CS-US paired trials followed by one CS-alone trial. Trials were separated by a variable intertrial interval pseudorandomized between 20 and $40 \mathrm{~s}$, with a mean of $30 \mathrm{~s}$. The acquisition session was continued until the average frequency of adaptive CRs over three consecutive sessions reached $>60 \%$, or for a maximum of $10 \mathrm{~d}$. Forty-six rats exhibited an average frequency of adaptive CRs over $3 \mathrm{~d}$ of $>60 \%$ within $9 \mathrm{~d}$ of acquisition $(65.9 \pm 2.4 \%)$. The other 51 rats did not reach the criterion and received $10 \mathrm{~d}$ of the acquisition sessions. Among these, 27 rats exhibited CRs of $>35 \%$ (51.2 $\pm 3.4 \%)$ and were used for additional experiments, but 24 rats whose frequency of CR was $<35 \%$ were excluded from additional study. Seventy-three rats in total were divided into six groups: the PBS group $(n=6)$ and the muscimol group $(n=6)$ for the experiment of acute inactivation, and the aCSF group ( $n=6$ for 1 d group, $n=7$ for 1 week group, $n=9$ for 2 week group, and $n=7$ for second 2 week group) and the APV group ( $n=$ 6 for 1 d group, $n=7$ for 1 week group, $n=11$ for 2 week group, and $n=$ 8 for second 2 week group) for the chronic infusion experiment. Six weeks after the end of the initial training, the rats were conditioned again using the trace paradigm for $7 \mathrm{~d}$ to test their memory retention. All experiments were performed during the light phase of the light/dark cycle.

EMG analysis. The method of analysis of the EMG data was the same as in our previous study (Takehara et al., 2003). Briefly, the mean + SD of the EMG amplitudes during the pre-CS period (0-300 ms before CS onset) of 100 trials was defined as the threshold. In each trial, the average values of EMG amplitudes above the threshold were calculated for 300 $\mathrm{ms}$ before the CS onset (prevalue), for all sets of the $100 \mathrm{~ms}$ period of EMG data between $50 \mathrm{~ms}$ after the CS onset and the US onset (CR value), and for $200 \mathrm{~ms}$ before the US onset (adaptive CR value). We discarded trials in which the prevalue exceeded $10 \%$ of threshold. A trial was assumed to contain a $C R$ or adaptive $C R$ if its $C R$ value or adaptive $C R$ value exceeded $10 \%$ of the threshold, respectively. The ratio of the number of trials containing the adaptive CR to the total number of valid trials was denoted the adaptive CR percentage for each session. To show the temporal pattern of the CR, the EMG amplitude data for each rat were 
averaged over the valid trials for each day. These trial-averaged EMG amplitude data were normalized by the time-averaged values for $300 \mathrm{~ms}$ before the CS onset. The CR onset and peak latencies were calculated from all the trials that were judged to contain a CR.

In vivo electrophysiology. Twenty-five rats were used for the in vivo electrophysiological study. We implanted the rats with the same type of cannulas used with the osmotic pumps in the behavioral experiment, with the following coordinates: $\mathrm{AP}+4.2, \mathrm{ML}+0.8$ to bregma, and $\mathrm{DV}$ -3.2 to the skull surface. After a recovery period of 7-9 d, the rats were anesthetized with $1 \mathrm{~g} / \mathrm{kg}$ urethane and $25 \mathrm{mg} / \mathrm{kg} \alpha$-chloralose (i.p.) and fixed in a stereotaxic frame. To record field EPSPs (fEPSPs), a tungsten recording electrode was inserted into the prelimbic area of the $\mathrm{MPFC}$ (AP +3.2, $\mathrm{ML}+0.8, \mathrm{DV}-3.2$ ), and bipolar stainless-steel stimulating electrodes were placed in the ventral CA1/subicular region of the hippocampus (AP - 6.0, ML +5.6, DV -6.8). The positions of the two electrodes were adjusted until a stable fEPSP was recorded. Constant-current test stimulation ( $80 \mu$ s duration) was applied at intervals of $30 \mathrm{~s}$, and its intensity was adjusted to produce an fEPSP with a slope that was $\approx 50 \%$ of maximum. To induce long-term potentiation (LTP), high-frequency burst stimulation (HFBS) consisting of 10 bursts (each with 50 pulses at $250 \mathrm{~Hz}$ ) at $0.1 \mathrm{~Hz}$ (at the same stimulation intensity that we used for the test stimuli) was delivered to the ventral CA1/subicular region. We presented this pattern of stimuli three times every $3 \mathrm{~min}$. The slope of the fEPSP was measured at a maximum slope of $1 \mathrm{~ms}$ duration. In part of the experiment, paired-pulse facilitation was observed by applying pairedpulse stimulation at an interpulse interval of $50 \mathrm{~ms}$. After the electrophysiological experiments, an electric lesion was made to confirm the position of the recording electrode.

Histology. After termination of the experimental procedure, each animal was intraperitoneally injected with an excess amount of sodium pentobarbital (>80 mg/kg; Dainippon Pharmaceutical, Osaka, Japan) and perfused intracardially with $0.9 \%$ saline, followed by phosphatebuffered $10 \%$ formalin. The brain was removed from the skull and stored in $10 \%$ formalin for a few days. After infiltration with $30 \%$ sucrose, the brain was frozen, sectioned at $60 \mu \mathrm{m}$, and stained with cresyl violet. The sections were subsequently examined under a light microscope and the locations of the cannula tips and the recording electrodes were drawn onto plates from the stereotaxic atlas of the rat brain (Paxinos and Watson, 1986). The distance between the electrode tip and the infusion cannula was estimated by counting the number of sections between them.

Statistical analysis. All data are expressed as the mean \pm SEM. Statistical significance was determined by a two-way ANOVA with repeated measures or by a $t$ test using SPSS statistical software (SPSS Japan, Tokyo, Japan). A value of $p<0.05$ was regarded as significant.

\section{Results}

Reversible inactivation of the $\mathrm{mPFC}$ during reconditioning severely impairs retrieval of remotely acquired memory.

First, we investigated whether reversible inactivation of the rostral $\mathrm{mPFC}$ centering in the prelimbic area during reconditioning can replicate the severe impairment in retrieval (retention) of remotely acquired memory, which we reported previously by using a permanent lesion method (Takehara et al., 2003). Figure 1 shows the effect of acute microinfusion of muscimol into the $\mathrm{mPFC}$ on retrieval of remotely acquired memory. Six weeks after the end of the initial conditioning, the rats received reconditioning, which is the same protocol we used in our previous study (Takehara et al., 2003). The rats in the muscimol group (muscimol, $n=6$ ) received microinfusions of muscimol in sessions 1 and 3, and microinfusions of PBS in session 2. The muscimol infusion into the mPFC almost completely abolished the adaptive CR in sessions 1 and 3. However, the same rats could express adaptive CRs when they received PBS infusion in session 2. Statistical analysis using a two-way repeated measures ANOVA on sessions $1-3$ of the reconditioning revealed that there was a significant interaction between groups and sessions $\left(F_{(2,20)}=\right.$

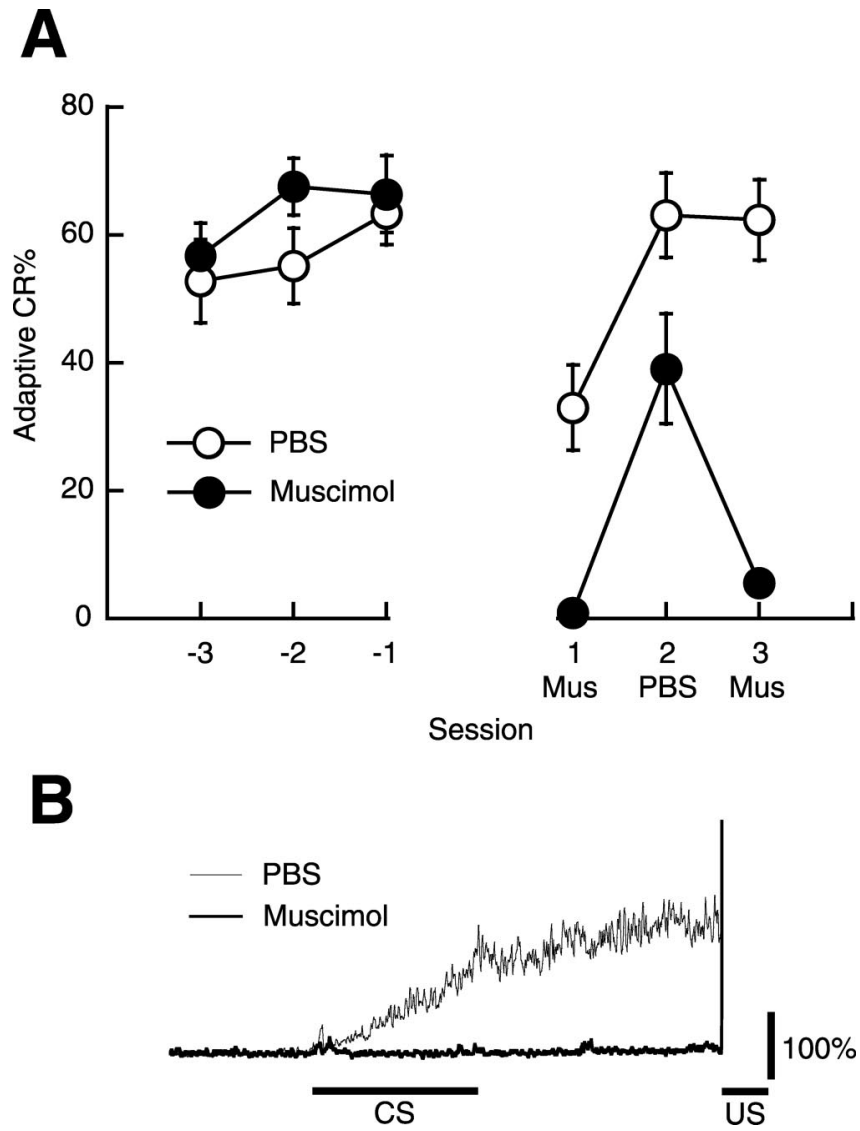

Figure 1. Acute inactivation of the $\mathrm{MPFC}$ completely abolishes retrieval of remotely acquired memory. A, Mean percentage of adaptive CRs during the last $3 \mathrm{~d}$ of the initial conditioning and reconditioning. Negative values on the abscissa represent the last $3 \mathrm{~d}$ of the initial conditioning (the session in which the rat reached the criterion is denoted -1 ), and positive values represent the reconditioning. During reconditioning, the muscimol group (muscimol, $n=6$; filled circles) exhibited significantly fewer adaptive CRs in sessions 1 and 3 when it received the muscimol infusion than did the PBS group (PBS, $n=6$; open circles), although the same group could exhibit comparable adaptive (Rs in session 2 when it received PBS infusion. $\boldsymbol{B}$, Averaged EMG amplitude of the PBS group (thin line) and the muscimol group (thick line) in session 1. The solid line under each trace indicates the timing of the $350 \mathrm{~ms}$ CS and the $100 \mathrm{~ms}$ US. The vertical scale indicates the average EMG amplitude before the CS presentation. The EMG amplitude of the muscimol group showed little increase after the $C S$ onset.

3.96; $p<0.05)$. The adaptive CR percentage of the muscimol group was significantly different from that of the PBS group in sessions 1 and 3 (two-tailed $t$ test: $t_{5.02}=4.81, p<0.01 ; t_{5.45}=$ $8.89, p<0.001$, respectively) and that the adaptive CR percentage of the muscimol group in session 2 was not different from that of the PBS group in session 1 (two-tailed $t$ test: $t_{10}=-0.56, p>$ $0.05)$. We also confirmed the lack of CRs in the muscimol group by investigating the averaged EMG amplitude over all the valid trials in session 1 (Fig. $1 B$ ). The EMG amplitude of the muscimol-infusion group was almost flat after the CS onset. These results suggest that the rostral mPFC centering on the prelimbic area is important for retrieval of remotely acquired memory. Thus, we set out to investigate the effect of chronic blockade of NMDA receptors in this area on the process through which this area gradually gains importance for memory.

\section{NMDA receptor blockade during the first 2 weeks of the postlearning period severely impairs memory retention measured 6 weeks after learning.}

We examined the effect of NMDA receptor blockade in the mPFC during the first 2 weeks of the postlearning period on memory 


\section{A}

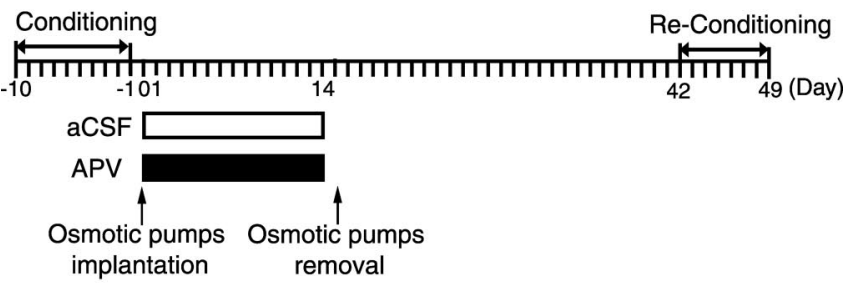

B

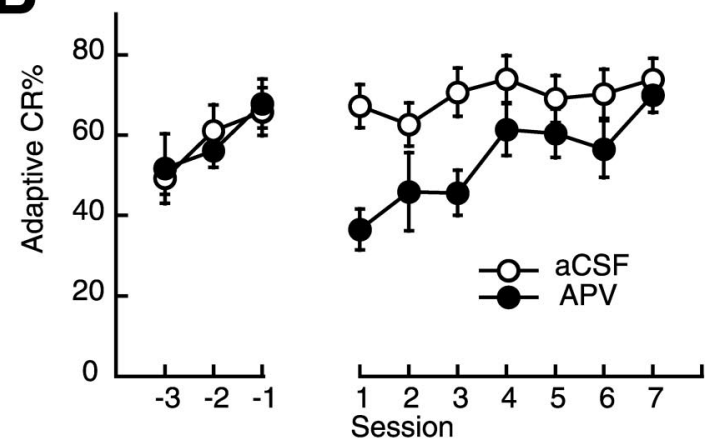

C

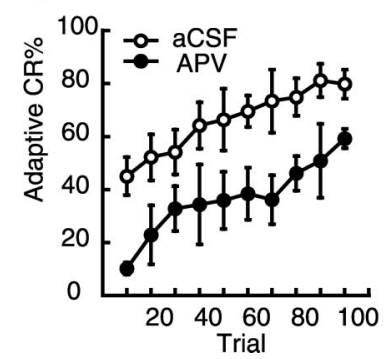

D

Figure 2. Chronic blockade of NMDA receptors in the $\mathrm{MPFC}$ during the first 2 weeks of the postlearning period prevents retention of remotely acquired memory. $A$, Experimental design. The rats received chronic infusion of D-APV $(n=7)$ or aCSF $(n=8)$ during the initial 2 weeks of the postlearning period. Their memory retention was measured 6 weeks after conditioning when memory depends critically on the mPFC. $\boldsymbol{B}$, Mean percentage of adaptive CRs during the last $3 \mathrm{~d}$ of the initial conditioning and reconditioning. Negative values on the abscissa represent the last $3 \mathrm{~d}$ of the initial conditioning (the session in which the rat reached the criterion is denoted -1 ), and positive values represent the reconditioning. During reconditioning, the APV group (filled circles) showed a significantly lower adaptive CR percentage in the first session, although it could relearn the adaptive (R up to the level of the aCSF group (empty circles) with subsequent retraining. C, Mean percentage of adaptive CRs during session 1 divided into 10 blocks. The daily 100 trials were divided into 10 blocks. The APV group (filled circles) consistently exhibited a lower adaptive CR percentage than the aCSF group (empty circles) during session 1. D, Averaged EMG amplitude of the aCSF group (thin line) and the APV group (thick line) in session 1. The solid line under each trace indicates the timing of the $350 \mathrm{~ms} C S$ and the $100 \mathrm{~ms}$ US. The vertical scale indicates the average EMG amplitude before the $C S$ presentation. The temporal pattern of CRs in the APV group exhibited no notable change that could explain the impairment observed in this group.

retention at 6 weeks after learning (i.e., the end of the conditioning) in trace eyeblink conditioning. The experimental schedule is shown in Figure $2 \mathrm{~A}$. During reconditioning, the rats that received the D-APV infusion during the first 2 weeks of the 6 week postlearning period (APV group, $n=7$ ) exhibited severely impaired adaptive CR retention compared with those infused with aCSF (aCSF group, $n=8$ ) (Fig. $2 B$ ). Statistical analysis using a two-way repeated measures ANOVA on sessions 1-7 revealed that there was no significant interaction between groups and sessions $\left(\mathrm{F}_{(6,78)}=1.61 ; p>0.05\right)$, but there were significant effects of groups $\left(\mathrm{F}_{(1,13)}=9.06 ; p<0.05\right)$ and sessions $\left(\mathrm{F}_{(6,78)}=3.92 ; p<\right.$
0.001). The impairment observed in the APV group was especially prominent in session 1 , but this group gradually relearned the adaptive CR up to the level of the control group with subsequent retraining. This observation was supported by our finding of a significant difference between the aCSF and APV groups on session $1\left(t_{13}=4.12 ; p<0.001\right)$ but no significant difference on session $7\left(t_{13}=0.56 ; p>0.05\right)$ using a two-tailed $t$ test. When we divided the 100 trials in session 1 into 10 blocks, the APV group consistently exhibited a lower adaptive CR percentage than the aCSF group (two-way repeated measures ANOVA; groups by sessions: $\mathrm{F}_{(9,108)}=0.43, p>0.05$; groups: $\mathrm{F}_{(1,12)}=26.7, p<$ 0.001; sessions: $\left.\mathrm{F}_{(9,108)}=6.87, p<0.001\right)$ (Fig. $2 C$ ).

To examine the drug effect on the temporal pattern of the CR, we investigated the averaged EMG amplitude over all the valid trials in session 1 (Fig. 2D). Consistent with the lower adaptive CR percentage in the APV groups, their average EMG amplitude was smaller than that of the aCSF group. To quantify the change in the temporal pattern of the CR, we measured the CR onset and peak latencies (all CRs observed after the CS onset were included). The CR onset latency of the APV group (370.1 \pm 41.4 $\mathrm{ms}$ ) was not significantly different from that of the aCSF group $\left(262.2 \pm 32.8 \mathrm{~ms}\right.$ ) (two-tailed $t$ test; $\left.t_{13}=-2.07, p>0.05\right)$. However, the CR peak latency of the APV group $(565.0 \pm 22.8$ ms) was significantly longer than that of the aCSF group $(478.6 .1 \pm 5.9 \mathrm{~ms})$ (two-tailed $t$ test: $t_{13}=-3.90, p<0.001$ ), suggesting that the EMG amplitude of the APV group increases to its maximum closer to the US onset than the aCSF group's amplitude does. This difference suggests that the residual CRs observed in the APV group are more adaptive to avoid the US than those in the aCSF group. Thus, the observed impairment is not attributable to abnormal response timing. These results suggest that activation of NMDA receptors in the MPFC during the first 2 weeks of the post-training period is necessary for successful memory retention 6 weeks after learning.

\section{NMDA receptor blockade during the second 2 weeks of the postlearning period has no effect on memory retention at 6 weeks after learning.}

Next, we moved the infusion period to the second 2 weeks of the postlearning period (from 2 weeks to 4 weeks after the end of the conditioning) (Fig. 3A). Again, 6 weeks after learning, the rats were retrained to measure their retention of the adaptive CR. The rats that received the D-APV infusion (APV group, $n=7$ ) exhibited an adaptive $\mathrm{CR}$ retention comparable with that of those infused with aCSF (aCSF, $n=7$ ) from the first day of the retraining (Fig. 3B). Statistical analysis using a two-way repeated measures ANOVA on sessions 1-7 revealed that there was no significant interaction between groups and sessions $\left(F_{(6,72)}=1.71 ; p>0.05\right)$ and no significant effect of groups $\left(F_{(1,12)}=0.76 ; p>0.05\right)$, but there was a significant session effect $\left(F_{(6,72)}=6.72 ; p<0.001\right)$. When we divided the 100 trials in session 1 into 10 blocks, we confirmed the lack of difference between groups in session 1 (two-way repeated measures ANOVA; groups by sessions: $F_{(9,108)}=1.28, p>0.05$; groups: $F_{(1,12)}=0.80, p>0.05$; sessions: $\left.F_{(9,108)}=11.7, p<0.001\right)$ (Fig. $\left.3 C\right)$. The tendency for the aCSF group in this experiment to show a relatively lower adaptive CR percentage in session 1 compared with the aCSF group receiving infusion during the first 2 weeks may be attributable to insufficient recovery from two closely scheduled surgeries for the removal of pumps and implantation of the EMG electrodes (Fig. $3 A$ ). In addition, the absence of the effect of the APV cannot be attributed to a ceiling effect because there was no statistically significant difference between the APV group that received infu- 


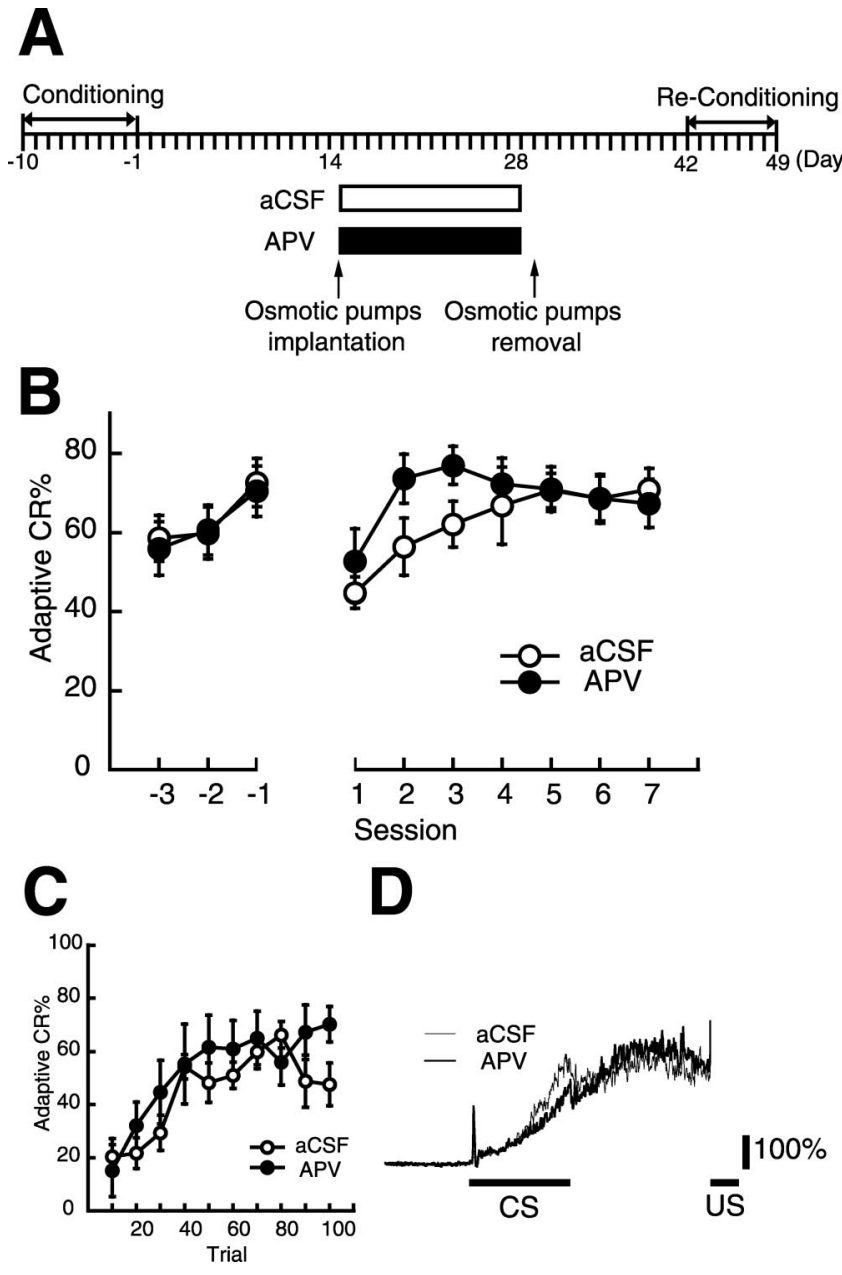

Figure 3. Chronic blockade of NMDA receptors in the MPFC during the second 2 weeks of the postlearning period has no effect on retention of remotely acquired memory. $\boldsymbol{A}$, Experimental design. The rats received chronic infusion of D-APV $(n=7)$ or aCSF $(n=7)$ during the second 2 weeks of the postlearning period. Their memory retention was measured 6 weeks after training when the memory depends critically on the MPFC. $\boldsymbol{B}$, Mean percentage of adaptive CRs during the last $3 \mathrm{~d}$ of the initial conditioning and reconditioning. Negative values on the abscissa represent the last $3 \mathrm{~d}$ of the initial conditioning (the session in which the rat reached the criterion is denoted -1 ), and positive values represent the reconditioning. During the reconditioning, the APV group (filled circles) could exhibit an adaptive CR percentage comparable with the aCSF group (empty circles). C, Mean percentage of adaptive CRs during session 1 divided into 10 blocks. The daily 100 trials were divided into 10 blocks. The performance during session 1 was not different between groups (APV group, filled circles; aCSF group, empty cir(les). $D$, Averaged EMG amplitude of the aCSF group (thin line) and the APV group (thick line) in session 1. The solid line under each trace indicates the timing of the $350 \mathrm{~ms}$ CS and the $100 \mathrm{~ms}$ US. The vertical scale indicates the average EMG amplitude before the CS presentation. There were no obvious differences in the temporal pattern of the CRs among the groups.

sion during the second 2 weeks of the post-training period and the aCSF group receiving infusion during the first 2 weeks (twoway repeated measures ANOVA; groups by sessions: $F_{(6,78)}=$ $1.70, p>0.05$; groups: $F_{(1,13)}=0.02, p>0.05$; sessions: $F_{(6,78)}=$ 2.05, $p>0.05)$.

We also investigated the averaged EMG amplitude over all the valid trials in session 1 and found that the EMG trace of the APV group was very similar to that of the aCSF group (Fig. $3 D$ ). To analyze the change in the temporal pattern of the CR quantitatively, we measured the CR onset and peak latencies (all CRs observed after the CS onset were included). Neither the CR onset latency nor the CR peak latency differed between the groups [the CR onset latency: $319.1 \pm 33.2 \mathrm{~ms}$ (aCSF group), $278.1 \pm 48.1 \mathrm{~ms}$
(APV group), two-tailed $t$ test: $t_{12}=0.70, p>0.05$; the CR peak latency: $519.6 \pm 25.6 \mathrm{~ms}$ (aCSF group), $562.6 \pm 21.8 \mathrm{~ms}$ (APV group), two-tailed $t$ test: $\left.t_{12}=-1.28, p>0.05\right]$. These results suggest that activation of NMDA receptors in the $\mathrm{mPFC}$ during the second 2 weeks of the post-training period is not necessary for successful memory retention 6 weeks after learning.

\section{NMDA receptor blockade during the first week, but not during the first day of the postlearning period impairs memory retention measured 6 weeks after learning.}

To further specify the critical time window when the NMDA receptors in the $\mathrm{mPFC}$ are important, we narrowed the period of NMDA receptor blockade in the $\mathrm{mPFC}$ to the first day or the first week of the postlearning period. Figure 4 shows the effect of NMDA receptor blockade in the $\mathrm{mPFC}$ during the first week of the postlearning period on memory retention at 6 weeks after learning (Fig. 4A). During reconditioning, the rats that had received the D-APV infusion during the first week of the 6 week postlearning period (APV group, $n=7$ ) exhibited a tendency toward impairment in adaptive $\mathrm{CR}$ retention compared with those infused with aCSF (aCSF group, $n=6$ ) (Fig. $4 B$ ). Statistical analysis using a two-way repeated measures ANOVA on sessions 1-7 revealed that there was no significant interaction between groups and sessions $\left(F_{(6,66)}=1.27 ; p>0.05\right)$ but there were significant session effects $\left(F_{(6,66)}=4.31 ; p<0.01\right)$, although the group effects did not reach statistical significance $\left(F_{(1,11)}=3.58\right.$; $p=0.085)$. The impairment observed in the APV group was especially prominent in session 1 , but this group gradually relearned the adaptive CR up to the level of the control group with subsequent retraining. This observation was supported by our finding of a significant difference between the aCSF and APV groups on session $1\left(t_{11}=2.26 ; p<0.05\right)$, but no significant difference on session $7\left(t_{11}=0.47 ; p>0.05\right)$ using a two-tailed $t$ test. When we divided the 100 trials in session 1 into 10 blocks, the APV group consistently exhibited a lower adaptive CR percentage than the aCSF group (two-way repeated measures ANOVA; groups by sessions: $F_{(9,99)}=0.62, p>0.05$; groups: $F_{(1,11)}=4.97$, $p<0.05$; sessions: $F_{(9,99)}=5.22, p<0.001$ ) (Fig. $4 C$ ). To examine the drug effect on the temporal pattern of the CR, we investigated the averaged EMG amplitude over all the valid trials in session 1 (Fig. 4D). Consistent with the lower adaptive CR percentage in the APV groups, their average EMG amplitude was also smaller than that of the aCSF group. To quantify the change in the temporal pattern of the CR, we measured the CR onset and peak latencies (all CRs observed after the CS onset were included). Neither the CR onset latency nor the CR peak latency differed between the groups [the CR onset latency: $289.0 \pm 33.6$ ms (aCSF group), $334.0 \pm 44.5 \mathrm{~ms}$ (APV group), two-tailed $t$ test: $t_{11}=-0.78, p>0.05$; the CR peak latency: $485.4 \pm 16.8 \mathrm{~ms}$ (aCSF group), $540.1 \pm 25.0 \mathrm{~ms}$ (APV group), two-tailed $t$ test: $\left.t_{11}=-0.78, p>0.05\right]$. Thus, the observed impairment is not attributable to abnormal response timing. These results suggest that blockade of NMDA receptors in the mPFC during the first week of the post-training period can produce impairments in memory retention 6 weeks after learning that are similar to those seen during the first 2 weeks of the post-training period.

Figure 5 shows the effect of NMDA receptor blockade in the $\mathrm{mPFC}$ during the first day of the postlearning period on memory retention at 6 weeks after learning (Fig. 5A). The rats that received the D-APV infusion (APV group, $n=6$ ) exhibited an adaptive CR retention comparable with that of those infused with aCSF (aCSF, $n=6$ ) on the first day of the retraining and thereafter (Fig. $5 B)$. Statistical analysis using a two-way repeated measures 

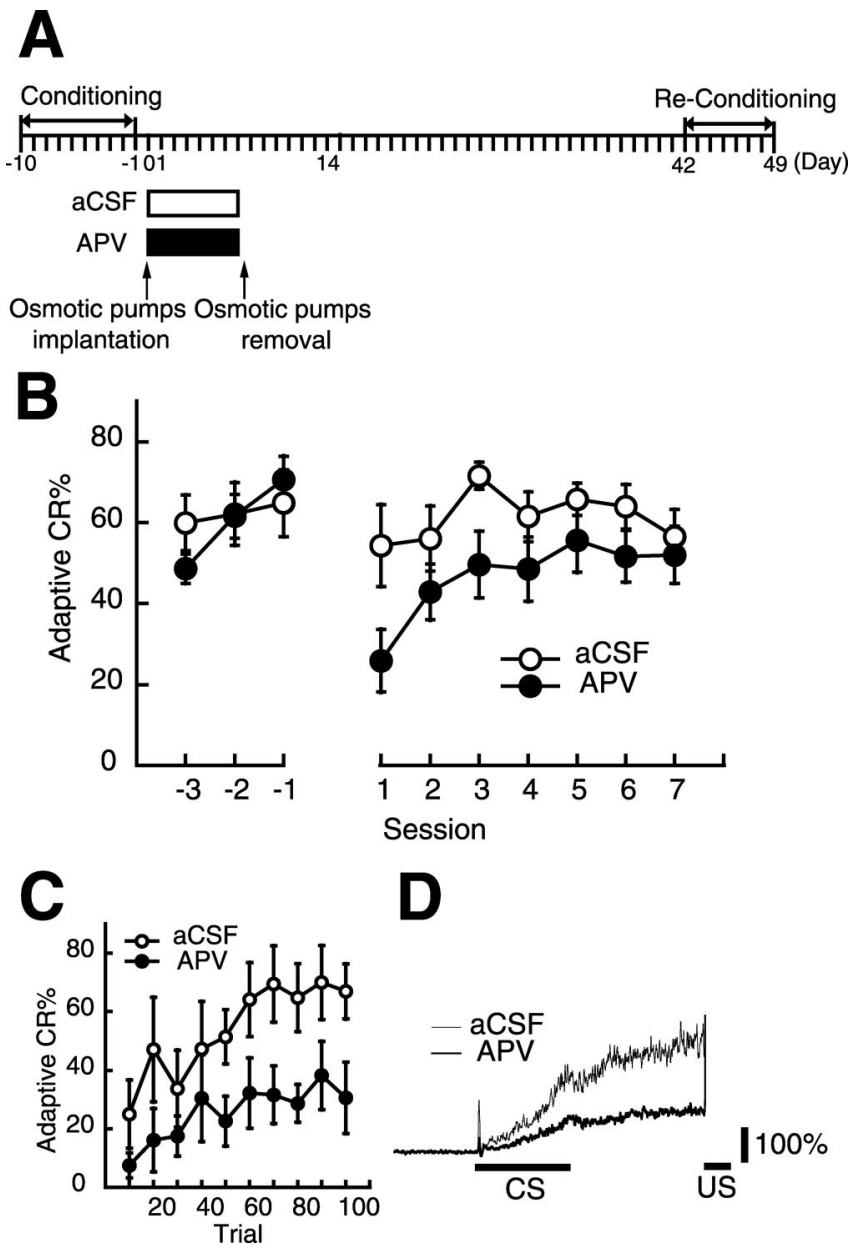

Figure 4. Chronic blockade of NMDA receptors in the MPFC during the first week of the postlearning period prevents retention of remotely acquired memory. $A$, Experimental design. The rats received chronic infusion of $D$-APV $(n=7)$ or aCSF $(n=6)$ during the initial week of the postlearning period. Their memory retention was measured 6 weeks after conditioning when memory depends critically on the mPFC. $\boldsymbol{B}$, Mean percentage of adaptive CRs during the last $3 \mathrm{~d}$ of the initial conditioning and reconditioning. Negative values on the abscissa represent the last $3 \mathrm{~d}$ of the initial conditioning (the session in which the rat reached the criterion is denoted -1 ) and positive values represent the reconditioning. During reconditioning, the APV group (filled circles) showed a significantly lower adaptive CR percentage in the first session, although it could relearn the adaptive (R up to the level of the aCSF group (empty circles) with subsequent retraining. C, Mean percentage of adaptive CRs during session 1 divided into 10 blocks. The daily 100 trials were divided into 10 blocks. The APV group (filled circles) consistently exhibited a lower adaptive CR percentage than the aCSF group (empty circles) during session 1.D. Averaged EMG amplitude of the aCSF group (thin line) and the APV group (thick line) in session 1. The solid line under each trace indicates the timing of the $350 \mathrm{~ms} C S$ and the $100 \mathrm{~ms}$ US. The vertical scale indicates the average EMG amplitude before the $C S$ presentation. The temporal pattern of CRs in the APV group exhibited no notable change that could explain the impairment observed in this group.

ANOVA on sessions 1-7 revealed that there was no significant interaction between groups and sessions $\left(F_{(6,60)}=0.59 ; p>0.05\right)$ and no significant effect of groups $\left(F_{(1,10)}=1.85 ; p>0.05\right)$, but there was a significant effect of session $\left(F_{(6,60)}=3.22 ; p<0.01\right)$. When we divided the 100 trials in session 1 into 10 blocks, we confirmed the lack of difference between groups in session 1 (two-way repeated measures ANOVA; groups by sessions: $F_{(9,90)}=$ $0.91, p>0.05$; groups: $F_{(1,10)}=0.25, p>0.05$; sessions: $F_{(9,90)}=$ 13.1, $p<0.001$ ) (Fig. 5C). We also investigated the averaged EMG amplitude over all the valid trials in session 1 and found that the EMG trace of the APV group was very similar to that of
A

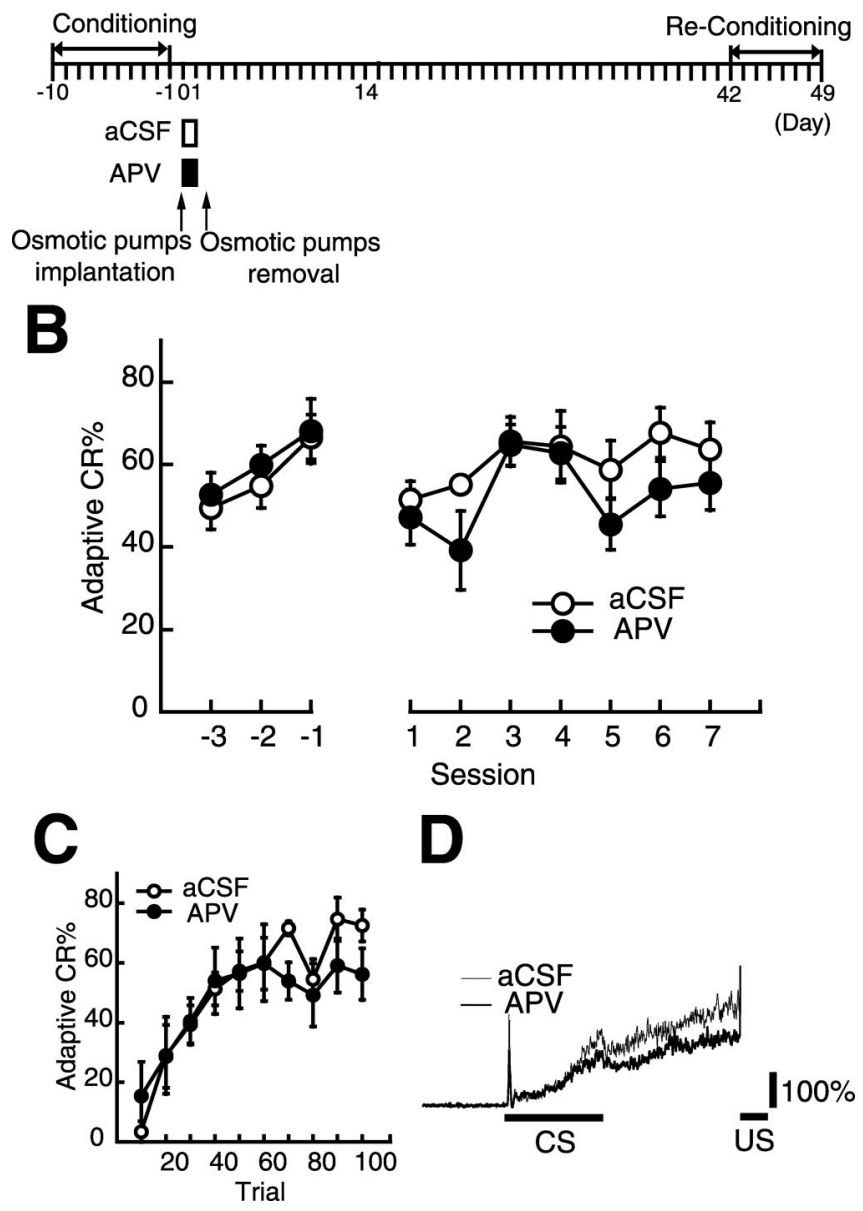

Figure 5. Chronic blockade of NMDA receptors in the mPFC during the first day of the postlearning period has no effect on retention of remotely acquired memory. $A$, Experimental design. The rats received chronic infusion of D-APV $(n=7)$ or aCSF $(n=6)$ during the first day of the postlearning period. Their memory retention was measured 6 weeks after training when the memory depends critically on the mPFC. $\boldsymbol{B}$, Mean percentage of adaptive CRs during the last $3 \mathrm{~d}$ of the initial conditioning and reconditioning. Negative values on the abscissa represent the last $3 \mathrm{~d}$ of the initial conditioning (the session in which the rat reached the criterion is denoted -1 ) and positive values represent the reconditioning. During the reconditioning, the APV group (filled circles) could exhibit an adaptive CR percentage comparable with the aCSF group (empty circles). C, Mean percentage of adaptive (Rs during session 1 divided into 10 blocks. The daily 100 trials were divided into 10 blocks. The performance during session 1 did not differ between groups (APV group, filled circles; aCSF group, empty circles). D, Averaged EMG amplitude of the aCSF group (thin line) and the APV group (thick line) in session 1. The solid line under each trace indicates the timing of the $350 \mathrm{~ms}$ CS and the $100 \mathrm{~ms} U \mathrm{US}$. The vertical scale indicates the average EMG amplitude before the CS presentation. There were no obvious differences in the temporal pattern of the CRs among the groups.

the aCSF group (Fig. 5D). To analyze the change in the temporal pattern of the CR quantitatively, we measured the CR onset and peak latencies (all CRs observed after the CS onset were included). Neither the CR onset latency nor the CR peak latency differed between the groups [the CR onset latency: $321.6 \pm 31.9$ $\mathrm{ms}$ (aCSF group), $327.8 \pm 48.4 \mathrm{~ms}$ (APV group), two-tailed $t$ test: $t_{10}=-0.11, p>0.05$; the CR peak latency: $529.4 \pm 11.3 \mathrm{~ms}$ (aCSF group), $546.7 \pm 27.0 \mathrm{~ms}$ (APV group), two-tailed $t$ test: $\left.t_{10}=-0.60, p>0.05\right]$. These results suggest that NMDA blockade in the MPFC during the first day of the post-training period is not sufficient to impair memory retention 6 weeks after learning. 


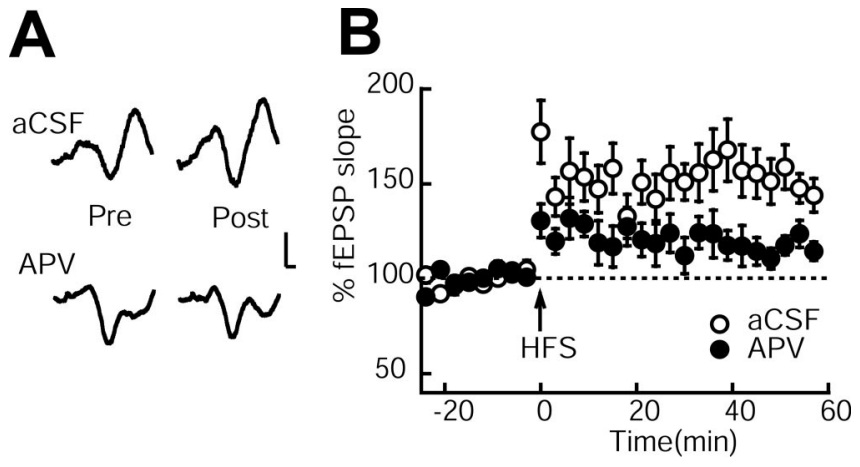

Figure 6. The NMDA receptor antagonist D-APV blocks LTP in the hippocampal-prefrontal cortex pathway. $\boldsymbol{A}$, Typical field potentials immediately before (pre) and after (post) HFBS application. Calibration: $0.1 \mathrm{mV}, 5 \mathrm{~ms}$. $\boldsymbol{B}$, Time course of changes in fEPSP slopes in anesthetized rats treated with aCSF (empty circles) and rats treated with D-APV (filled circles). HFBS was applied to the $\mathrm{CA} 1$ /ventral subicular area at time $0 \mathrm{~min}$. All data points represent group mean \pm SEM of 6-10 animals.

\section{Chronic infusion of D-APV attenuated NMDA receptor- dependent LTP in the hippocampal-prefrontal pathway.} The extent of the blockade of NMDA receptors in the mPFC was examined electrophysiologically. Because the direct projection from the CA1/ventral subicular region to the prelimbic area of the $\mathrm{MPFC}$ is endowed with NMDA receptor-dependent LTP (Laroche et al., 1990; Jay et al., 1995), we measured this in vivo LTP in the rats that received chronic infusion of aCSF (aCSF group, $n=$ 10 ) or D-APV (APV group, $n=6$ ) with an osmotic pump in the same procedure as the above behavioral experiments (Fig. 6). The baseline electrophysiological responses evoked over a range of stimulus intensities before tetanization were not significantly different between the two groups; the averages of the fEPSP slopes after single-pulse stimulation at a stimulus intensity of $400 \mu \mathrm{A}$ were $40.4 \pm 6.7 \mu \mathrm{V} / \mathrm{ms}$ (aCSF group) and $48.1 \pm 8.2 \mu \mathrm{V} / \mathrm{ms}$ (APV group), and at an intensity of $600 \mu \mathrm{A}$ they were $45.4 \pm 1.1$ $\mu \mathrm{V} / \mathrm{ms}$ (aCSF group) and $53.1 \pm 7.2 \mu \mathrm{V} / \mathrm{ms}$ (APV group) (twotailed $t$ test: $t_{14}=-0.70, p>0.05$ and $t_{14}=-0.51, p>0.05$, respectively).

When HFBS was applied to the ventral CA1/subicular region, the fEPSPs of the aCSF group immediately increased, and homosynaptic LTP was induced. In contrast, the fEPSP of the APV group increased after the same application of HFBS, but the magnitude of the LTP was smaller than in the aCSF group (Fig. 6). The average percent changes in the fEPSP slopes 30-60 min after HFBS stimulation were $155.1 \pm 10.4 \%$ in the aCSF group and $108.9 \pm 11.3 \%$ in the APV group (two-tailed $t$ test: $t_{14}=2.34, p<$ $0.05)$. To examine whether APV significantly affects the presynaptic or postsynaptic mechanism, we observed paired-pulse facilitation in the hippocampal-prefrontal pathway. The paired-pulse protocol (interpulse interval, $50 \mathrm{~ms}$ ) produced responses that were statistically indistinguishable between the aCSF group (140.2 $\pm 11.3 \%)$ and the APV group (125.4 $\pm 12.6 \%)$ (two-tailed $t$ test: $\left.t_{14}=0.84, p>0.05\right)$, indicating that APV has little effect on presynaptic responses in the mPFC. Thus, chronic infusion of APV attenuated the induction of LTP in the hippocampalprefrontal pathway without affecting basal synaptic transmission.

\section{Histology}

We examined the locations of the cannula tips and electrodes of all the rats after completion of the behavioral and electrophysiological studies. Figure $7 A$ shows a representative photomicro- graph of a brain section of a rat used in the experiment of chronic infusion. By examining Nissl-stained sections in a blinded manner, we verified that the cannula placements were within the appropriate area of the prelimbic cortex and that damage to the tissue was limited to just around the cannula. Seven of 73 rats were rejected using this criterion, leaving 12 rats for the groups that received acute infusion (acute groups), 12 rats for the group that received infusion during the first day of the postlearning period ( $1 \mathrm{~d}$ group), 13 rats for the group that received infusion during the first week of the postlearning period (1 week group), 15 rats for the groups that received infusion during the first 2 weeks of the postlearning period (2 week group) and 14 rats for the groups that received infusion during the second 2 weeks (second 2 week group). In the two rats in the 2 week APV group, the cannula was placed dorsally in the anterior cingulate area, and in another rat the cannula in the right side is located ventrolateral to the ventral orbital area, although the cannula in the left side is located in the prelimbic area. These rats showed little decline in the adaptive CR percentage in the first retraining session. These results suggest that APV infusion mainly into the rostral anterior cingulate area or unilaterally into the prelimbic area was not sufficient to impair memory retention 6 weeks after the learning. After the electrophysiological experiment, we determined again, in a blinded manner, that the electrode tips were within the appropriate area of the prelimbic region and the distance between the cannula tip and electrodes was never $>2 \mathrm{~mm}$. Four of 14 rats in the aCSF group and one of eight rats in the APV group were rejected by this criterion. One rat in the APV group exhibited an abnormal EPSP waveform, and therefore the data from this rat were discarded, leaving 6 rats in the APV group. Figure $7 B$ depicts the cannula tip placements in the rats included in the above analysis ( $B$, the acute group; $C$, the $1 \mathrm{~d}$ groups; $D$, the 1 week groups; $E$, the 2 week groups; $F$, the second 2 week groups; $G$, the rats in the electrophysiological study). In all cases, the cannula placements of the experimental groups were not different from those of the control groups.

\section{Discussion}

Our previous results showing severe impairment in retention of remotely acquired memory after permanent lesions of the mPFC was confirmed by the reversible inactivation experiment. Next, we investigated whether specific manipulation of neuronal processes in the mPFC affects successful establishment of remote memory. To block neuronal processes in the mPFC, we used osmotic pumps for chronic focal infusion of the NMDA receptor antagonist D-APV. The hippocampal-prefrontal pathway of the rats with chronic infusion of D-APV displayed reduced LTP without any notable change in basal synaptic transmission. Chronic blockade of NMDA receptors in the mPFC during the first 1 or 2 weeks of the postlearning period severely impaired memory retention 6 weeks after learning, but the same treatment during the first day or second 2 weeks had no effect on memory retention. These results suggest that the integrity of the NMDA receptor system in the mPFC is necessary for systems consolidation through which memory is gradually stabilized in the neocortical network.

\section{The MPFC as a critical region for systems consolidation}

Because we blocked NMDA receptors specifically during the consolidation period without affecting any processes during acquisition and retrieval, the straightforward interpretation of the present results is that some changes occur in the $\mathrm{mPFC}$ during the initial week of the postlearning period, and that these changes are 
necessary for successful establishment of remotely acquired memory. However, there are several other possibilities to be considered. (1) One might argue that chronic infusion of the NMDA receptor antagonist for weeks produced irreversible mPFC dysfunction, and disruption of the operation of mPFCs during retraining produced impaired memory retention. However, this possibility is unlikely because the same chronic infusion had no effect on memory retention if it was performed during the second 2 weeks of the postlearning period (Fig. 3B). (2) Although we chronically infused D-APV to affect systems consolidation, there exists the possibility that interference with cellular consolidation, rather than systems consolidation, caused the impairments we observed in the present experiment. However, the fact that the involvement of NMDA receptors in the $\mathrm{MPFC}$ in cellular consolidation is limited to the time when a memory is beginning to be formed (Takehara-Nishiuchi et al., 2005) leads us to reject this possibility. Therefore, the present results suggest that neural processes in the MPFC are required for systems consolidation. If one begins with the idea that information contained within the hippocampus directs consolidation by gradually changing the organization of cortical representations that store permanent memory (McClelland et al., 1995; Squire and Alvarez, 1995), then failure to retain remote memory by blockade of neural processes during consolidation is a prerequisite for a brain area to play an essential role in networks that store permanent memory. Together with our previous results showing that permanent lesion of the mPFC selectively impairs retention of remote memory (Takehara et al., 2003), which has now been replicated by the present data on reversible inactivation, the present results provide strong support for the notion that the $\mathrm{mPFC}$ is a critical component of the brain network that stores permanent memory. The importance of the $\mathrm{mPFC}$ in processing remote memory has been confirmed by studies of other learning tasks (Bontempi et al., 1999; Frankland et al., 2004; Maviel et al., 2004). Because the $\mathrm{mPFC}$ is connected to a wide range of neocortical and subcortical areas (Kolb, 1984), the mPFC possesses traits suitable for maintaining coherence among widely distributed memory modules (Wiltgen et al., 2004; Frankland and Bontempi, 2005). Therefore, the mPFC may communicate with other brain areas and integrate consolidation processes in several neocortical and subcortical areas, and may come to act as a critical component of the network that supports permanent memory storage. However, previous studies have suggested the importance of the PFC for several cognitive functions, such as working memory, top-down attention, and cognitive control (Fuster, 2001; Miller and Cohen, 2001; Uylings et al., 2003). It is an open question whether the function we propose for the rat mPFC in long-term memory consolidation and storage is a novel function of this
A

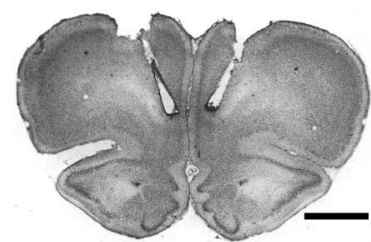

○ aCSF

C

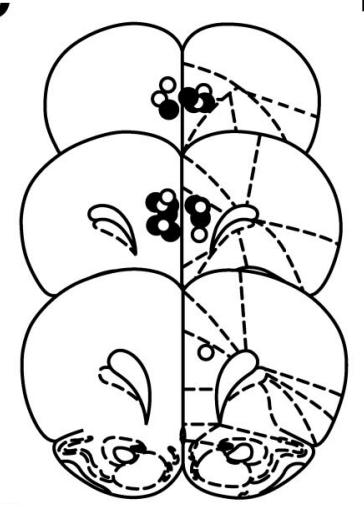

D

- APV

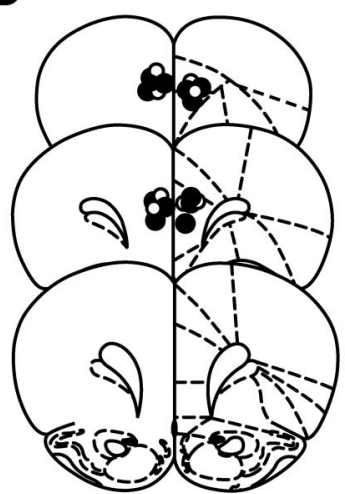

$F$

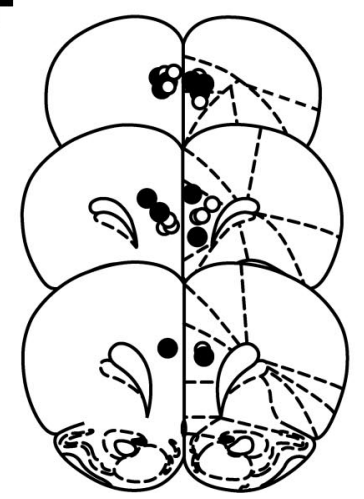

$\mathbf{G}$

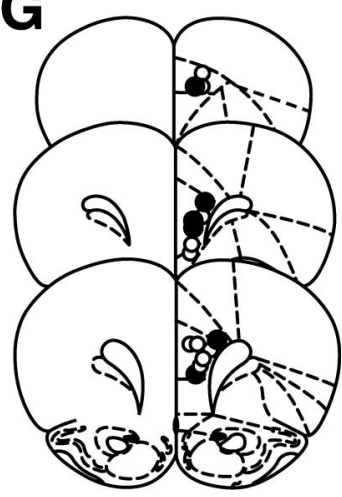

Figure 7. Histological reconstructions of cannula placements. $\boldsymbol{A}$, A representative photomicrograph of a coronal section from a rat with chronic infusion of APV. Scale bar, $2 \mathrm{~mm}$. B-G, Schematic illustration of the location of cannula tips in the rats' mPFCs, the rats in the electrophysiological study $(\boldsymbol{G})$. An empty or filled circle represents a placement in the aCSF or APV group, respectively. Numbers to the left indicate stereotaxic coordinates relative to bregma (Paxinos and Watson, 1986).

area, or can be interpreted as an extension of these previously proposed functions of the PFC.

\section{Time course of cortical consolidation}

We found that NMDA receptors in the mPFC are important during at least the initial week of the postlearning period. This time window is consistent with another report that the activation of $\alpha$-calcium/calmodulin-dependent protein kinase II (CaMKII) (the downstream target of NMDA receptor mediated signals) in the forebrain during the first post-training week is necessary for consolidation of fear memories (Wang et al., 2003). Similar to our time window, knock-out of the NR1 subunit of the NMDA receptor in the CA1 region of the hippocampus (Shimizu et al., 2000) or reversible inactivation of the hippocampus (Riedel et al., 1999) can each effectively block consolidation if performed during the initial week. These findings suggest the possibility that the neocortex and the hippocampus activate within a similar time window and that specific information is transferred between these two areas which directs neocortical reorganization during the initial postlearning week. This idea is supported by coherent reactivation of memory traces within the neocortex and the hip- 
pocampus during the postlearning period (Qin et al., 1997; Hoffman and McNaughton, 2002; Ribeiro et al., 2004).

\section{NMDA receptor-dependent mechanisms of systems consolidation}

Using the selective NMDA receptor antagonist D-APV, we revealed the importance of NMDA receptors in the consolidation processes that occur in the mPFC. Our in vivo electrophysiological data showing specific impairment in the LTP in the hippocampal-mPFC pathway suggest that the impairments observed in the behavioral study can be attributed primarily to blockade of NMDA receptors in the MPFC, rather than to nonspecific blockade of other receptors such as AMPA receptors. Because NMDA receptors are involved in many important physiological processes, there are several possible roles in systems consolidation for NMDA receptors in the mPFC. (1) NMDA receptor-mediated LTP in the hippocampal-prefrontal pathway was significantly reduced in our rats (Fig. 6). Therefore, there is a possibility that this direct projection from the hippocampus to the mPFC serves as the main pathway for transfer of information that directs neocortical reorganization. This pathway is activated during a critical phase of consolidation, as suggested by a delayed increase in synaptic transmission and by learning-induced changes in the expression of genes associated with plasticity in this pathway (for review, see Laroche et al. 2000). It has been reported that neurons in the $\mathrm{MPFC}$ are phase-locked to the hippocampal theta rhythm with lags averaging $50 \mathrm{~ms}$ (Siapas et al., 2005), suggesting that the hippocampus could entrain neuronal activity in the mPFC through this direct pathway. NMDA receptor-dependent plasticity in this pathway can strengthen the hippocampal modulation of neurons in the $\mathrm{MPFC}$ and may promote maturation of neural networks in the mPFC. (2) It is possible and even likely that alterations in synaptic plasticity are not restricted to the hippocampal-prefrontal pathway; they may also occur in connections from other neocortical areas to the mPFC in the rats infused with D-APV. During the course of systems consolidation, the strengthening of corticocortical connections is presumed to be crucial in allowing a neocortical memory network to gain independence from the hippocampus (McClelland et al., 1995; Squire and Alvarez, 1995). Thus, the present result suggests the possibility that NMDA receptor-dependent plasticity at cortico-to-mPFC connections is necessary for this maturation process. Similarly, it had been proposed that multiple rounds of NMDA receptor-dependent synaptic modification can reinforce the previously initiated synaptic changes and serve as a cellular means for strengthening intercortical connections (Shimizu et al., 2000; Wang et al., 2003). These possibilities are supported by the fact that mutant mice that are heterozygous for the null mutation of $\alpha$-CaMKII exhibit impaired cortical LTP and deficient retention of remote memory (Frankland et al., 2001). In addition, NMDA receptors play a critical role in activity-dependent dendritic growth (Wong and Ghosh, 2002). Thus, it is also possible that activation of NMDA receptors can trigger structural changes in the connections between neocortical neurons in the mPFC (Chklovskii et al., 2004), which is supported by a report that transgenic mice with altered cortical synaptic morphology exhibit specific impairment in retention of remote memory (Hayashi et al., 2004). (3) NMDA receptors are involved in the stimulus-specific persistent neural activity observed in the mPFC (Wang, 2001; Fellous and Sejnowski, 2003). We speculate that such persistent activity is also induced by the input from the hippocampus during the reactivation of memory modules that is presumed to occur during systems consolidation
(Sutherland and McNaughton, 2000), and that such persistent activity facilitates reorganization of corticocortical connections.

In conclusion, the present study suggests that the mPFC plays a critical role in the consolidation of remote memory through its NMDA receptor-dependent processes and that the importance of these processes is greatest during the first 2 weeks of the postlearning period in trace eyeblink conditioning. Additional studies using neuronal ensemble recordings will be able to clarify what aspect of memory is stored in the neuronal assemblies of the mPFC.

\section{References}

Bontempi B, Laurent-Demir C, Destrade C, Jaffard R (1999) Timedependent reorganization of brain circuitry underlying long-term memory storage. Nature 400:671-675.

Chklovskii DB, Mel BW, Svoboda K (2004) Cortical rewiring and information storage. Nature 431:782-788.

Debiec J, LeDoux JE, Nader K (2002) Cellular and systems reconsolidation in the hippocampus. Neuron 36:527-538.

Fellous JM, Sejnowski TJ (2003) Regulation of persistent activity by background inhibition in an in vitro model of a cortical microcircuit. Cereb Cortex 13:1232-1241.

Frankland PW, Bontempi B (2005) The organization of recent and remote memories. Nat Rev Neurosci 6:119-130.

Frankland PW, O’Brien C, Ohno M, Kirkwood A, Silva AJ (2001) AlphaCaMKII-dependent plasticity in the cortex is required for permanent memory. Nature 411:309-313.

Frankland PW, Bontempi B, Talton LE, Kaczmarek L, Silva AJ (2004) The involvement of the anterior cingulate cortex in remote contextual fear memory. Science 304:881-883.

Fuster JM (2001) The prefrontal cortex - an update: time is of the essence. Neuron 30:319-333.

Hayashi ML, Choi SY, Rao BS, Jung HY, Lee HK, Zhang D, Chattarji S, Kirkwood A, Tonegawa S (2004) Altered cortical synaptic morphology and impaired memory consolidation in forebrain-specific dominantnegative PAK transgenic mice. Neuron 42:773-787.

Hirsch JC, Crepel F (1991) Blockade of NMDA receptors unmasks a longterm depression in synaptic efficacy in rat prefrontal neurons in vitro. Exp Brain Res 85:621-624.

Hoffman KL, McNaughton BL (2002) Coordinated reactivation of distributed memory traces in primate neocortex. Science 297:2070-2073.

Jay TM, Burette F, Laroche S (1995) NMDA receptor-dependent long-term potentiation in the hippocampal afferent fibre system to the prefrontal cortex in the rat. Eur J Neurosci 7:247-250.

Kim JJ, Clark RE, Thompson RF (1995) Hippocampectomy impairs the memory of recently, but not remotely, acquired trace eyeblink conditioned responses. Behav Neurosci 109:195-203.

Knowlton BJ, Fanselow MS (1998) The hippocampus, consolidation and on-line memory. Curr Opin Neurobiol 8:293-296.

Kolb B (1984) Functions of the frontal cortex of the rat: a comparative review. Brain Res Rev 8:65-98.

Laroche S, Jay TM, Thierry AM (1990) Long-term potentiation in the prefrontal cortex following stimulation of the hippocampal CA1/subicular region. Neurosci Lett 114:184-190.

Laroche S, Davis S, Jay TM (2000) Plasticity at hippocampal to prefrontal cortex synapses: dual roles in working memory and consolidation. Hippocampus 10:438-446.

Maviel T, Durkin TP, Menzaghi F, Bontempi B (2004) Sites of neocortical reorganization critical for remote spatial memory. Science 305:96-99.

McClelland JL, McNaughton BL, O’Reilly RC (1995) Why there are complementary learning systems in the hippocampus and neocortex: insights from the successes and failures of connectionist models of learning and memory. Psychol Rev 102:419-457.

Miller EK, Cohen JD (2001) An integrative theory of prefrontal cortex function. Annu Rev Neurosci 24:167-202.

Nadel L, Moscovitch M (1997) Memory consolidation, retrograde amnesia and the hippocampal complex. Curr Opin Neurobiol 7:217-227.

Paxinos G, Watson C (1986) The rat brain in stereotaxic coordinates. San Diego: Academic.

Qin YL, McNaughton BL, Skaggs WE, Barnes CA (1997) Memory repro- 
cessing in corticocortical and hippocampocortical neuronal ensembles. Philos Trans R Soc Lond B Biol Sci 352:1525-1533.

Ribeiro S, Gervasoni D, Soares ES, Zhou Y, Lin SC, Pantoja J, Lavine M, Nicolelis MA (2004) Long-lasting novelty-induced neuronal reverberation during slow-wave sleep in multiple forebrain areas. PLoS Biol 2:E24.

Riedel G, Micheau J, Lam AG, Roloff E, Martin SJ, Bridge H, Hoz L, Poeschel B, McCulloch J, Morris RG (1999) Reversible neural inactivation reveals hippocampal participation in several memory processes. Nat Neurosci 2:898-905.

Shimizu E, Tang YP, Rampon C, Tsien JZ (2000) NMDA receptordependent synaptic reinforcement as a crucial process for memory consolidation. Science 290:1170-1174.

Siapas AG, Lubenov EV, Wilson MA (2005) Prefrontal phase locking to hippocampal theta oscillations. Neuron 46:141-151.

Squire LR (1992) Memory and the hippocampus: a synthesis from findings with rats, monkeys, and humans. Psychol Rev 99:195-231.

Squire LR, Alvarez P (1995) Retrograde amnesia and memory consolidation: a neurobiological perspective. Curr Opin Neurobiol 5:169-177.

Sutherland GR, McNaughton B (2000) Memory trace reactivation in hippocampal and neocortical neuronal ensembles. Curr Opin Neurobiol 10:180-186.

Takehara K, Kawahara S, Takatsuki K, Kirino Y (2002) Time-limited role of the hippocampus in the memory for trace eyeblink conditioning in mice. Brain Res 951:183-190.
Takehara K, Kawahara S, Kirino Y (2003) Time-dependent reorganization of the brain components underlying memory retention in trace eyeblink conditioning. J Neurosci 23:9897-9905.

Takehara-Nishiuchi K, Kawahara S, Kirino Y (2005) NMDA receptordependent processes in the medial prefrontal cortex are important for acquisition and the early stage of consolidation during trace but not delay eyeblink conditioning. Learn Mem 12:606-614.

Thompson RF, Kim JJ (1996) Memory systems in the brain and localization of a memory. Proc Natl Acad Sci USA 93:13438-13444.

Uylings HB, Groenewegen HJ, Kolb B (2003) Do rats have a prefrontal cortex? Behav Brain Res 146:3-17.

Wang H, Shimizu E, Tang YP, Cho M, Kyin M, Zuo W, Robinson DA, Alaimo PJ, Zhang C, Morimoto H, Zhuo M, Feng R, Shokat KM, Tsien JZ (2003) Inducible protein knockout reveals temporal requirement of CaMKII reactivation for memory consolidation in the brain. Proc Natl Acad Sci USA 100:4287-4292.

Wang XJ (2001) Synaptic reverberation underlying mnemonic persistent activity. Trends Neurosci 24:455-463.

Wiltgen BJ, Brown RA, Talton LE, Silva AJ (2004) New circuits for old memories: the role of the neocortex in consolidation. Neuron 44:101-108.

Wong RO, Ghosh A (2002) Activity-dependent regulation of dendritic growth and patterning. Nat Rev Neurosci 3:803-812. 